\title{
ON THIRD-ORDER LINEAR DIFFERENCE EQUATIONS INVOLVING QUASI-DIFFERENCES
}

ZUZANA DOŠLÁ AND ALEŠ KOBZA

Received 30 June 2004; Revised 20 September 2004; Accepted 12 October 2004

We study the third-order linear difference equation with quasi-differences and its adjoint equation. The main results of the paper describe relationships between the oscillatory and nonoscillatory solutions of both equations.

Copyright (c) 2006 Hindawi Publishing Corporation. All rights reserved.

\section{Introduction}

Consider the third-order linear difference equation

$$
\Delta\left(p_{n} \Delta\left(r_{n} \Delta x_{n}\right)\right)+q_{n} x_{n+1}=0
$$

and its adjoint equation

$$
\Delta\left(r_{n+1} \Delta\left(p_{n} \Delta u_{n}\right)\right)-q_{n+1} u_{n+2}=0,
$$

where $\Delta$ is the forward difference operator defined by $\Delta x_{n}=x_{n+1}-x_{n},\left(p_{n}\right),\left(r_{n}\right)$, and $\left(q_{n}\right)$ are sequences of positive real numbers for $n \in \mathbb{N}$.

This paper has been motivated by the paper [9], where third-order difference equations

$$
\begin{gathered}
\Delta^{3} v_{n}-p_{n+1} \Delta v_{n+1}+q_{n+1} v_{n+1}=0, \\
\Delta\left(\Delta^{2} u_{n}-p_{n+1} u_{n+1}\right)-q_{n+2} u_{n+2}=0
\end{gathered}
$$

had been investigated. As it is noted here, these equations are not adjoint equations and are referred to as quasi-adjoint equations.

Equation (E) is a special case of linear $n$ th-order difference equations with quasi-differences. Such equations have been widely studied in the literature, see, for example, $[6,11]$ and the references therein. The natural question which arises is to find the adjoint equation to (E) and to examine the connection between solutions of (E) and its adjoint one. 
In the continuous case, it holds (see, e.g., [5, Theorem 8.33]) that

$$
\left(\frac{1}{p(t)}\left(\frac{1}{r(t)} x^{\prime}(t)\right)^{\prime}\right)^{\prime}+q(t) x(t)=0
$$

is oscillatory if and only if the adjoint equation

$$
\left(\frac{1}{r(t)}\left(\frac{1}{p(t)} x^{\prime}(t)\right)^{\prime}\right)^{\prime}-q(t) x(t)=0
$$

has the same property. In addition, nonoscillatory solutions of these equations satisfy some interesting relationships, see, for example, $[2,5]$.

The aim of this paper is to investigate oscillatory and asymptotic properties of solutions of $(\mathrm{E})$ and $\left(\mathrm{E}^{A}\right)$. We will prove that $\left(\mathrm{E}^{A}\right)$ is the adjoint equation to $(\mathrm{E})$ and we will give discrete analogues of the above-quoted results for third-order differential equations. Moreover, the oscillation of $(\mathrm{E})$ and $\left(\mathrm{E}^{A}\right)$ is characterized by means of second-order linear difference equations and the problem of the number of oscillatory solutions in a given basis for the solution space of $(\mathrm{E})$ and $\left(\mathrm{E}^{A}\right)$ is investigated. Our results extend and complete results of [7-10] stated for the various forms of third-order difference equations.

A solution $x$ of (E) is a real sequence $\left(x_{n}\right)$ defined for all $n \in \mathbb{N}$ and satisfying (E) for all $n \in \mathbb{N}$. A solution of (E) is called nontrivial if for any $n_{0} \geq 1$, there exists $n>n_{0}$ such that $x_{n} \neq 0$. Otherwise, the solution is called trivial. A nontrivial solution $x$ of (E) is said to be oscillatory if for any $n_{0} \geq 1$, there exists $n>n_{0}$ such that $x_{n+1} x_{n} \leq 0$. Otherwise, the nontrivial solution is said to be nonoscillatory. Equation (E) is oscillatory if it has an oscillatory solution. The same terminology is used for $\left(\mathrm{E}^{A}\right)$.

Denote quasi-differences $x^{[i]}, i=0,1,2$, of a solution $x$ of $(\mathrm{E})$ as follows:

$$
x_{n}^{[0]}=x_{n}, \quad x_{n}^{[1]}=r_{n} \Delta x_{n}, \quad x_{n}^{[2]}=p_{n} \Delta x_{n}^{[1]}, \quad x_{n}^{[3]}=\Delta x_{n}^{[2]} .
$$

Similarly, denote quasi-differences $u^{[i]}, i=0,1,2$, of a solution $u$ of $\left(\mathrm{E}^{A}\right)$ as follows:

$$
u_{n}^{[0]}=u_{n}, \quad u_{n}^{[1]}=p_{n} \Delta u_{n}, \quad u_{n}^{[2]}=r_{n+1} \Delta u_{n}^{[1]}, \quad u_{n}^{[3]}=\Delta u_{n}^{[2]} .
$$

All nonoscillatory solutions $x$ of $(\mathrm{E})$ can be a priori classified to the following classes:

$$
\begin{aligned}
& N_{0}=\left\{x: \exists n_{x} \text { s.t. } x_{n} x_{n}^{[1]}<0, x_{n} x_{n}^{[2]}>0 \forall n \geq n_{x}\right\}, \\
& N_{1}=\left\{x: \exists n_{x} \text { s.t. } x_{n} x_{n}^{[1]}>0, x_{n} x_{n}^{[2]}<0 \forall n \geq n_{x}\right\}, \\
& N_{2}=\left\{x: \exists n_{x} \text { s.t. } x_{n} x_{n}^{[1]}>0, x_{n} x_{n}^{[2]}>0 \forall n \geq n_{x}\right\}, \\
& N_{3}=\left\{x: \exists n_{x} \text { s.t. } x_{n} x_{n}^{[1]}<0, x_{n} x_{n}^{[2]}<0 \forall n \geq n_{x}\right\},
\end{aligned}
$$

and similarly solutions $u$ of $\left(\mathrm{E}^{A}\right)$ can be classified to the same classes, whereby quasidifferences $u^{[i]}, i=1,2$, are defined by $(1.5)$, see $[4,3]$. Solutions of (E) from the class $N_{0}$ are called Kneser solutions and solutions of $\left(\mathrm{E}^{A}\right)$ which belong to the class $\mathrm{N}_{2}$ are called strongly monotone solutions. 


\section{Relationship between $(\mathrm{E})$ and $\left(\mathrm{E}^{A}\right)$}

Solutions of $(\mathrm{E})$ and $\left(\mathrm{E}^{A}\right)$ are related by the following properties.

Theorem 2.1. (a) Let $x, y$ be solutions of $(E)$. Then the sequence $C=\left(C_{n}\right)(n \geq 2)$ such that

$$
C_{n-1}=C\left(x_{n-1}, y_{n-1}\right) \equiv\left|\begin{array}{cc}
x_{n-1} & y_{n-1} \\
x_{n-1}^{[1]} & y_{n-1}^{[1]}
\end{array}\right|
$$

is a solution of $\left(E^{A}\right)$.

(b) Let $u, v$ be solutions of $\left(E^{A}\right)$. Then the sequence $D=\left(D_{n}\right)(n \geq 2)$ such that

$$
D_{n}=D\left(u_{n-1}, v_{n-1}\right) \equiv\left|\begin{array}{cc}
u_{n-1} & v_{n-1} \\
u_{n-1}^{[1]} & v_{n-1}^{[1]}
\end{array}\right|
$$

is a solution of $(E)$.

Proof. Claim (a). For any two solutions $x, y$ of (E), we have

$$
x_{n} \Delta y_{n-1}^{[2]}-y_{n} \Delta x_{n-1}^{[2]}=-x_{n} q_{n-1} y_{n}+y_{n} q_{n-1} x_{n}=0
$$

Therefore,

$$
\begin{aligned}
\Delta C_{n-1} & =x_{n} \Delta y_{n-1}^{[1]}+y_{n-1}^{[1]} \Delta x_{n-1}-y_{n} \Delta x_{n-1}^{[1]}-x_{n-1}^{[1]} \Delta y_{n-1} \\
& =x_{n} \Delta y_{n-1}^{[1]}+r_{n-1} \Delta y_{n-1} \Delta x_{n-1}-y_{n} \Delta x_{n-1}^{[1]}-r_{n-1} \Delta x_{n-1} \Delta y_{n-1} \\
& =x_{n} \Delta y_{n-1}^{[1]}-y_{n} \Delta x_{n-1}^{[1]} .
\end{aligned}
$$

Using the fact $x_{n-1}^{[2]}=x_{n}^{[2]}-\Delta x_{n-1}^{[2]}$ and (2.3), we obtain

$$
\begin{aligned}
C_{n-1}^{[1]} & =p_{n-1} \Delta C_{n-1}=x_{n} y_{n-1}^{[2]}-y_{n} x_{n-1}^{[2]} \\
& =x_{n}\left(y_{n}^{[2]}-\Delta y_{n-1}^{[2]}\right)-y_{n}\left(x_{n}^{[2]}-\Delta x_{n-1}^{[2]}\right)=x_{n} y_{n}^{[2]}-y_{n} x_{n}^{[2]} .
\end{aligned}
$$

By a direct computation in view of (2.3), we get

$$
\Delta C_{n-1}^{[1]}=x_{n+1} \Delta y_{n}^{[2]}+y_{n}^{[2]} \Delta x_{n}-y_{n+1} \Delta x_{n}^{[2]}-x_{n}^{[2]} \Delta y_{n}=y_{n}^{[2]} \Delta x_{n}-x_{n}^{[2]} \Delta y_{n}
$$

hence

$$
C_{n-1}^{[2]}=r_{n} \Delta C_{n-1}^{[1]}=x_{n}^{[1]} y_{n}^{[2]}-y_{n}^{[1]} x_{n}^{[2]} .
$$


4 Third-order linear difference equations

Finally

$$
\begin{aligned}
\Delta C_{n-1}^{[2]} & =x_{n+1}^{[1]} \Delta y_{n}^{[2]}+y_{n}^{[2]} \Delta x_{n}^{[1]}-y_{n+1}^{[1]} \Delta x_{n}^{[2]}-x_{n}^{[2]} \Delta y_{n}^{[1]} \\
& =-x_{n+1}^{[1]} q_{n} y_{n+1}+p_{n} \Delta y_{n}^{[1]} \Delta x_{n}^{[1]}+y_{n+1}^{[1]} q_{n} x_{n+1}-p_{n} \Delta x_{n}^{[1]} \Delta y_{n}^{[1]} \\
& =q_{n}\left(x_{n+1} y_{n+1}^{[1]}-y_{n+1} x_{n+1}^{[1]}\right)=q_{n} C_{n+1},
\end{aligned}
$$

that is, $C_{n-1}$ is a solution of $\left(\mathrm{E}^{A}\right)$.

Claim (b). By the similar argument as in (a), we get

$$
\Delta D_{n}=u_{n} \Delta v_{n-1}^{[1]}-v_{n} \Delta u_{n-1}^{[1]} .
$$

Using the fact $u_{n-1}^{[2]}=u_{n-2}^{[2]}+\Delta u_{n-2}^{[2]}$, we obtain

$$
\begin{aligned}
D_{n}^{[1]} & =r_{n} \Delta D_{n}=u_{n} v_{n-1}^{[2]}-v_{n} u_{n-1}^{[2]} \\
& =u_{n}\left(v_{n-2}^{[2]}+\Delta v_{n-2}^{[2]}\right)-v_{n}\left(u_{n-2}^{[2]}+\Delta u_{n-2}^{[2]}\right) \\
& =u_{n}\left(v_{n-2}^{[2]}+q_{n-1} v_{n}\right)-v_{n}\left(u_{n-2}^{[2]}+q_{n-1} u_{n}\right) \\
& =u_{n} v_{n-2}^{[2]}-v_{n} u_{n-2}^{[2]} .
\end{aligned}
$$

Using the same argument as before, we get

$$
\begin{aligned}
\Delta D_{n}^{[1]} & =v_{n-1}^{[2]} \Delta u_{n}+u_{n} \Delta v_{n-2}^{[2]}-u_{n-1}^{[2]} \Delta v_{n}-v_{n} \Delta u_{n-2}^{[2]} \\
& =v_{n-1}^{[2]} \Delta u_{n}-u_{n-1}^{[2]} \Delta v_{n} .
\end{aligned}
$$

Hence

$$
D_{n}^{[2]}=p_{n} \Delta D_{n}^{[1]}=u_{n}^{[1]} v_{n-1}^{[2]}-v_{n}^{[1]} u_{n-1}^{[2]} .
$$

Finally

$$
\begin{aligned}
\Delta D_{n}^{[2]} & =u_{n}^{[1]} \Delta v_{n-1}^{[2]}+v_{n}^{[2]} \Delta u_{n}^{[1]}-v_{n}^{[1]} \Delta u_{n-1}^{[2]}-u_{n}^{[2]} \Delta v_{n}^{[1]} \\
& =u_{n}^{[1]} q_{n} v_{n+1}+r_{n+1} \Delta v_{n}^{[1]} \Delta u_{n}^{[1]}-v_{n}^{[1]} q_{n} u_{n+1}-r_{n+1} \Delta u_{n}^{[1]} \Delta v_{n}^{[1]} \\
& =-q_{n}\left[v_{n}^{[1]}\left(\Delta u_{n}+u_{n}\right)-u_{n}^{[1]}\left(\Delta v_{n}+v_{n}\right)\right] \\
& =-q_{n}\left(p_{n} \Delta v_{n} \Delta u_{n}+u_{n} v_{n}^{[1]}-p_{n} \Delta u_{n} \Delta v_{n}-v_{n} u_{n}^{[1]}\right)=-q_{n} D_{n+1}
\end{aligned}
$$

that is, $D_{n}$ is a solution of $(\mathrm{E})$.

Relationship between solutions of $(\mathrm{E})$ and $\left(\mathrm{E}^{A}\right)$ described in Theorem 2.1 is a discrete analogue of the relationship valid for the differential (1.2) and its adjoint (1.3). For this reason, we call $\left(\mathrm{E}^{A}\right)$ the adjoint equation to $(\mathrm{E})$. This is in accordance with the definition of the adjoint system to the difference system as the following remark shows. 
Remark 2.2. According to [1, page 60], if $X=\left\{X_{n}\right\}$ is a nontrivial solution of the system

$$
X_{n+1}=A_{n} X_{n}
$$

then $U=\left\{U_{n}\right\}$, where $U_{n}=\left(X_{n}^{T}\right)^{-1}$ is a solution of the system

$$
U_{n}=A_{n}^{T} U_{n+1}
$$

System (2.15) is called the adjoint system of (2.14).

Equation (E) can be written as a first-order difference system

$$
\begin{aligned}
& \Delta x_{n}^{[0]}=\frac{1}{r_{n}} x_{n}^{[1]}, \\
& \Delta x_{n}^{[1]}=\frac{1}{p_{n}} x_{n}^{[2]}, \\
& \Delta x_{n}^{[2]}=-q_{n} x_{n+1}^{[0]},
\end{aligned}
$$

for the vector $X_{n}=\left(x_{n}^{[0]}, x_{n}^{[1]}, x_{n}^{[2]}\right)$. Since $x_{n+1}^{[0]}=x_{n}^{[0]}+\Delta x_{n}^{[0]}$, we have

$$
\Delta x_{n}^{[2]}=-q_{n}\left(x_{n}^{[0]}+\frac{1}{r_{n}} x_{n}^{[1]}\right) .
$$

Using the usual convention that no index actually means the index $n$, otherwise the index is explicitly specified, we obtain

$$
\left(\begin{array}{c}
l x_{n+1}^{[0]} \\
x_{n+1}^{[1]} \\
x_{n+1}^{[2]}
\end{array}\right)=\left(\begin{array}{ccc}
1 & \frac{1}{r} & 0 \\
0 & 1 & \frac{1}{p} \\
-q & -\frac{q}{r} & 1
\end{array}\right)\left(\begin{array}{c}
l x^{[0]} \\
x^{[1]} \\
x^{[2]}
\end{array}\right)
$$

Hence (E) can be interpreted as the system of the form (2.14). Its adjoint system is

$$
\left(\begin{array}{l}
l u^{[0]} \\
u^{[1]} \\
u^{[2]}
\end{array}\right)=\left(\begin{array}{ccc}
1 & 0 & -q \\
\frac{1}{r} & 1 & -\frac{q}{r} \\
0 & \frac{1}{p} & 1
\end{array}\right)\left(\begin{array}{c}
l u_{n+1}^{[0]} \\
u_{n+1}^{[1]} \\
u_{n+1}^{[2]}
\end{array}\right)
$$

From here we get

$$
\begin{aligned}
& \Delta u_{n}^{[0]}=q_{n} u_{n+1}^{[2]}, \\
& \Delta u_{n}^{[1]}=-\frac{1}{r_{n}} u_{n+1}^{[0]}+\frac{q_{n}}{r_{n}} u_{n+1}^{[2]}, \\
& \Delta u_{n}^{[2]}=-\frac{1}{p_{n}} u_{n+1}^{[1]},
\end{aligned}
$$


and the last equation gives $\Delta u_{n+1}^{[1]}=-\Delta\left(p_{n} \Delta u_{n}^{[2]}\right)$. Replacing the shift $n$ by $n+1$ and substituting into the second equation, we have

$$
-\Delta\left(p_{n} \Delta u_{n}^{[2]}\right)=-\frac{1}{r_{n+1}} u_{n+2}^{[0]}+\frac{q_{n+1}}{r_{n+1}} u_{n+2}^{[2]} .
$$

Multiplying this equation by $-r_{n+1}$ and differentiating it, we obtain

$$
\Delta\left(r_{n+1} \Delta\left(p_{n} \Delta u_{n}^{[2]}\right)\right)+\Delta\left(q_{n+1} u_{n+2}^{[2]}\right)=\Delta u_{n+2}^{[0]} .
$$

Substituting from the first equation in (2.20), we get

$$
\Delta\left(r_{n+1} \Delta\left(p_{n} \Delta u_{n}^{[2]}\right)\right)+q_{n+2} u_{n+3}^{[2]}-q_{n+1} u_{n+2}^{[2]}=q_{n+2} u_{n+3}^{[2]},
$$

which means that the sequence $v_{n}=u_{n}^{[2]}$ satisfies $\left(\mathrm{E}^{A}\right)$.

Notation 2.3. Let $S$ denote the solution space of (E) and let $S^{\star}$ denote the solution space of $\left(\mathrm{E}^{A}\right)$. For $(x, u) \in S \times S^{\star}$, define $\mathscr{L}=\left(\mathscr{L}_{n}\right)$, where

$$
\mathscr{L}_{n}=\mathscr{L}\left(x_{n}, u_{n}\right)=x_{n+1} u_{n}^{[2]}-x_{n+1}^{[1]} u_{n}^{[1]}+x_{n+1}^{[2]} u_{n+1} .
$$

The functional $\mathscr{L}$ has the following properties.

Lemma 2.4. The sequence $\mathscr{L}: S \times S^{\star} \rightarrow \mathbb{R}$ is a constant which depends only on the choice of solutions $x$ and $u$, and not on $n$.

Proof. By a direct computation we get

$$
\begin{aligned}
\Delta \mathscr{L}_{n}= & \Delta\left(x_{n+1} u_{n}^{[2]}-x_{n+1}^{[1]} u_{n}^{[1]}+x_{n+1}^{[2]} u_{n+1}\right) \\
= & x_{n+2} \Delta u_{n}^{[2]}+u_{n}^{[2]} \Delta x_{n+1}-x_{n+1}^{[1]} \Delta u_{n}^{[1]}-u_{n+1}^{[1]} \Delta x_{n+1}^{[1]} \\
& +u_{n+2} \Delta x_{n+1}^{[2]}+x_{n+1}^{[2]} \Delta u_{n+1} \\
= & x_{n+2} q_{n+1} u_{n+2}+r_{n+1} \Delta u_{n}^{[1]} \Delta x_{n+1}-r_{n+1} \Delta x_{n+1} \Delta u_{n}^{[1]} \\
& -p_{n+1} \Delta u_{n+1} \Delta x_{n+1}^{[1]}-u_{n+2} q_{n+1} x_{n+2}+p_{n+1} \Delta x_{n+1}^{[1]} \Delta u_{n+1}=0,
\end{aligned}
$$

which completes the proof.

Lemma 2.5. Let $x, y, z$ be solutions of (E). Let $C$ and $\mathscr{L}$ be defined by (2.1) and (2.24), respectively. Then the sequence $R=\left(R_{n}\right)$, where

$$
R_{n}=\left|\begin{array}{ccc}
x_{n} & y_{n} & z_{n} \\
x_{n}^{[1]} & y_{n}^{[1]} & z_{n}^{[1]} \\
x_{n}^{[2]} & y_{n}^{[2]} & z_{n}^{[2]}
\end{array}\right|
$$

satisfies

$$
R_{n}=\mathscr{L}\left(z_{n-1}, C_{n-1}\right)
$$


Proof. Expanding $R_{n}$ along its third column, we obtain

$$
R_{n}=z_{n}\left|\begin{array}{cc}
x_{n}^{[1]} & y_{n}^{[1]} \\
x_{n}^{[2]} & y_{n}^{[2]}
\end{array}\right|-z_{n}^{[1]}\left|\begin{array}{cc}
x_{n} & y_{n} \\
x_{n}^{[2]} & y_{n}^{[2]}
\end{array}\right|+z_{n}^{[2]}\left|\begin{array}{cc}
x_{n} & y_{n} \\
x_{n}^{[1]} & y_{n}^{[1]}
\end{array}\right| .
$$

Using (2.24), we have

$$
\mathscr{L}\left(z_{n-1}, C_{n-1}\right)=z_{n} C_{n-1}^{[2]}-z_{n}^{[1]} C_{n-1}^{[1]}+z_{n}^{[2]} C_{n} .
$$

From here, (2.1), (2.5), and (2.7) show that (2.27) holds.

\section{Nonoscillatory solutions of adjoint equations}

In this section, we study nonoscillatory solutions. We start with the following auxiliary results.

Lemma 3.1. There always exists nonoscillatory solution $u$ of $\left(E^{A}\right)$ with the property

$$
u_{n}>0, \quad u_{n}^{[1]}>0, \quad u_{n}^{[2]}>0 \quad \text { for } n \in \mathbb{N}
$$

that is, $\left(E^{A}\right)$ has a strongly monotone solution.

For the proof, see [4, Theorem 3.2].

LEMMA 3.2. If a solution $y$ of (E) satisfies for some integer $m>1$ that

$$
y_{m} \geq 0, \quad y_{m}^{[1]} \leq 0, \quad y_{m}^{[2]}>0,
$$

then

$$
y_{k}>0, \quad y_{k}^{[1]}<0, \quad y_{k}^{[2]}>0
$$

for each $k \in \mathbb{N}$ such that $1 \leq k<m$.

The proof follows from the proof of [3, Proposition 2].

The existence of Kneser solutions of (E) is ensured by the following result.

THeOREM 3.3. There always exists nonoscillatory solution $x$ of $(E)$ with the property

$$
x_{n}>0, \quad x_{n}^{[1]}<0, \quad x_{n}^{[2]}>0 \quad \text { for } n \in \mathbb{N},
$$

that is, (E) has a Kneser solution.

Proof. Let $x=(x(n)), y=(y(n)), z=(z(n))$ be a basis of the solution space $S$ of $(\mathrm{E})$. For $k \in \mathbb{N}$, define

$$
\omega_{k}(n)=a_{k} x(n)+b_{k} y(n)+c_{k} z(n)
$$

where $a_{k}, b_{k}, c_{k}$ are chosen such that

$$
\omega_{k}(k)=0, \quad \omega_{k}(k+1)=0, \quad a_{k}^{2}+b_{k}^{2}+c_{k}^{2}=1 .
$$


Then $\omega_{k}^{[1]}(k)=0$. By [3, Lemma 1], $\omega_{k}(k+2) \neq 0$. Without loss of generality, assume that $\omega_{k}(k+2)>0$. Then

$$
\omega_{k}^{[1]}(k+1)=r_{k+1} \Delta \omega_{k}(k+1)=r_{k+1}\left(\omega_{k}(k+2)-\omega_{k}(k+1)\right)>0,
$$

hence

$$
\omega_{k}^{[2]}(k)=p_{k} \Delta \omega_{k}^{[1]}(k)=p_{k}\left(\omega_{k}^{[1]}(k+1)-\omega_{k}^{[1]}(k)\right)>0 .
$$

Since

$$
\omega_{k}(k)=0, \quad \omega_{k}^{[1]}(k)=0, \quad \omega_{k}^{[2]}(k)>0,
$$

by Lemma 3.2

$$
\omega_{k}(n)>0, \quad \omega_{k}^{[1]}(n)<0, \quad \omega_{k}^{[2]}(n)>0, \quad \text { for } 1 \leq n<k .
$$

Put $A_{k}=\left(a_{k}, b_{k}, c_{k}\right)$. Then $\left\|A_{k}\right\|=1$ for each $k$. The unit ball is compact in $\mathbb{R}^{3}$, so $\left(A_{k}\right)$ has a convergent subsequence $\left(A_{k_{i}}\right)$. Denote

$$
A=\lim _{i \rightarrow \infty} A_{k_{i}}=(a, b, c) .
$$

Then $a^{2}+b^{2}+c^{2}=1$ and

$$
\omega(n)=\lim _{i \rightarrow \infty} \omega_{k_{i}}=\lim \left(a_{k_{i}} x(n)+b_{k_{i}} y(n)+c_{k_{i}} z(n)\right)
$$

is a nontrivial solution of (E). Then in view of (3.10) and the fact that $k$ is arbitrary integer, we get

$$
\omega(n) \geq 0, \quad \omega^{[1]}(n) \leq 0, \quad \omega^{[2]}(n) \geq 0 \quad \text { for } n \geq 1 .
$$

If $\omega\left(n_{0}\right)=0$ for some $n=n_{0}$, then $\omega(n)=0$ for all $n \geq n_{0}$ which is a contradiction with the fact that $\omega$ is a nontrivial solution. Thus $\omega(n)>0$ for every $n \geq 1$, and so

$$
\Delta\left(\omega^{[2]}(n)\right)=-q(n) \omega(n+1)<0 \quad \text { for } n \geq 1 .
$$

Hence, $\omega^{[2]}$ is decreasing and so $\omega^{[2]}(n)>0$ for $n \in \mathbb{N}$. From here $\Delta\left(\omega^{[1]}(n)\right)>0$ for $n \in$ $\mathbb{N}$, which implies that $\omega^{[1]}$ is increasing and $\omega^{[1]}(n)<0$ for $n \in \mathbb{N}$.

THEOREM 3.4. Every nonoscillatory solution of $\left(E^{A}\right)$ is strongly monotone if and only if every nonoscillatory solution of $(E)$ is a Kneser solution.

Proof. Let every nonoscillatory solution of $\left(\mathrm{E}^{A}\right)$ be strongly monotone. Assume by contradiction that there exists solution $y$ of (E) which belongs to the class $N_{i}$, where $i \in$ $\{1,2,3\}$. Let $x$ be a Kneser solution of (E). Without loss of generality, we may suppose that $x_{n}>0$ and $y_{n}>0$ for large $n$. Then the sequence $C$ defined by (2.1) is according to 
Theorem 2.1 solution of $\left(\mathrm{E}^{A}\right)$ and in view of (2.5) and (2.7) it satisfies, for large $n$,

$$
\begin{array}{lll}
C_{n-1}>0, & C_{n-1}^{[1]}<0 & (\text { if } i=1) \\
C_{n-1}>0, & C_{n-1}^{[2]}<0 & (\text { if } i=2) \\
C_{n-1}^{[1]}<0, & C_{n-1}^{[2]}>0 & (\text { if } i=3) .
\end{array}
$$

This is a contradiction with the fact that $C$ is strongly monotone solution.

Now suppose that every solution of $(\mathrm{E})$ is a Kneser solution. Assume by contradiction that there exists solution $v$ of $\left(\mathrm{E}^{A}\right)$ which belongs to the class $N_{i}$, where $i \in\{0,1,3\}$. Let $u$ be a strongly monotone solution of $\left(\mathrm{E}^{A}\right)$. Without loss of generality, we may suppose that $u_{n}>0$ and $v_{n}>0$ for large $n$. Then the sequence $D$ defined by (2.2) is according to Theorem 2.1 solution of (E) and it satisfies, for large $n$,

$$
\begin{array}{ccc}
D_{n}<0, & D_{n}^{[2]}>0 & (\text { if } i=0) \\
D_{n}^{[1]}<0, & D_{n}^{[2]}<0 & (\text { if } i=1) \\
D_{n}<0, & D_{n}^{[1]}<0 & (\text { if } i=3) .
\end{array}
$$

This is a contradiction with the fact that $D$ is a Kneser solution.

\section{Oscillatory properties of adjoint equations}

LEMMA 4.1. Let $u$ be a strongly monotone solution and $v$ an oscillatory solution of $\left(E^{A}\right)$. Then their Casoratian D defined by (2.2) is an oscillatory solution of (E).

Proof. By Theorem 2.1, $D$ is a solution of (E). We will show that $D$ is an oscillatory solution. Without loss of generality, we may suppose that $u$ satisfies (3.1). Since $v$ is an oscillatory solution, there exist increasing sequences of positive integers $\left(i_{n}\right)$ and $\left(j_{n}\right)$, with properties

$$
\begin{array}{lll}
v_{i_{n}} \leq 0, & v_{i_{n}}^{[1]}>0 & \text { for } n \in \mathbb{N}, \\
v_{j_{n}} \geq 0, & v_{j_{n}}^{[1]}<0 & \text { for } n \in \mathbb{N} .
\end{array}
$$

From the above inequalities, (2.2), and (3.1), we have

$$
D_{i_{n}+1}=u_{i_{n}} v_{i_{n}}^{[1]}-v_{i_{n}} u_{i_{n}}^{[1]}>0 \quad \text { for } n \in \mathbb{N},
$$

and similarly $D_{j_{n}+1}<0$ for $n \in \mathbb{N}$. Hence the sequence $D$ is an oscillatory solution of (E).

Lemma 4.2. Let $x$ be a Kneser solution and $y$ an oscillatory solution of $(E)$. Then their Casoratian $C$ defined by (2.1) is an oscillatory solution of $\left(E^{A}\right)$.

Proof. By Theorem 2.1, $C$ is a solution of $\left(\mathrm{E}^{A}\right)$. We will show that $C$ is an oscillatory solution. Without loss of generality, we may suppose that $x$ satisfies (3.4). Because $y$ is an oscillatory solution, there exist increasing sequences of positive integers $\left(i_{n}\right)_{1}^{\infty}$ and $\left(j_{n}\right)_{1}^{\infty}$ 
with properties

$$
\begin{array}{llll}
i_{1}>M, & y_{i_{n}} \leq 0, \quad y_{i_{n}}^{[1]}>0 & \text { for } n \in \mathbb{N}, \\
j_{1}>M, & y_{j_{n}} \geq 0, \quad y_{j_{n}}^{[1]}<0 & \text { for } n \in \mathbb{N},
\end{array}
$$

where $M=\min \left\{n \in \mathbb{N}: y_{n} y_{n+1} \leq 0\right\}$.

Assume that $y_{j_{n}}^{[2]}>0$ for some $n \in \mathbb{N}$. Then by Lemma 3.2, we get

$$
y_{k}>0, \quad y_{k}^{[1]}<0 \quad \text { for } 1 \leq k<j_{n}
$$

which is a contradiction with $j_{1}>M$. Hence $y_{j_{n}}^{[2]} \leq 0$ for $n \in \mathbb{N}$. From here and using (2.7) follows

$$
C_{j_{n}-1}^{[2]}=x_{j_{n}}^{[1]} y_{j_{n}}^{[2]}-y_{j_{n}}^{[1]} x_{j_{n}}^{[2]}>0 \quad \text { for } n \in \mathbb{N} \text {. }
$$

By similar argument as before, we obtain $y_{i_{n}}^{[2]} \geq 0$ for $n \in \mathbb{N}$, which implies that

$$
C_{i_{n}-1}^{[2]}=x_{i_{n}}^{[1]} y_{i_{n}}^{[2]}-y_{i_{n}}^{[1]} x_{i_{n}}^{[2]}<0 \quad \text { for } n \in \mathbb{N} .
$$

By [3, Lemma 2], it follows from inequalities (4.5) and (4.6) that $C$ is an oscillatory solution of $\left(\mathrm{E}^{A}\right)$. The proof is now complete.

Our next result characterizes the existence of oscillatory solutions of the adjoint equations.

Theorem 4.3. Equation $\left(E^{A}\right)$ is oscillatory if and only if $(E)$ is oscillatory.

The proof follows from Theorem 3.3 and Lemmas 3.1, 4.1, and 4.2.

In the sequel, we study the existence of an oscillatory solution in terms of second-order equations.

THEOREM 4.4. (a) If $u$ is a nonoscillatory solution of $\left(E^{A}\right)$, then two linearly independent solutions of $(E)$ satisfy the second-order difference equation

$$
p_{n+1} \Delta\left(\frac{r_{n+1} \Delta x_{n+1}}{u_{n+1}}\right)+\frac{u_{n}^{[2]}}{u_{n+1} u_{n+2}} x_{n+2}=0 .
$$

(b) If $x$ is a nonoscillatory solution of $(E)$, then two linearly independent solutions of $\left(E^{A}\right)$ satisfy the second-order difference equation

$$
r_{n+1} \Delta\left(\frac{p_{n} \Delta u_{n}}{x_{n+1}}\right)+\frac{x_{n+1}^{[2]}}{x_{n+1} x_{n+2}} u_{n+1}=0 .
$$

Proof. Claim (a). Let $u$ be a fixed nonoscillatory solution of $\left(\mathrm{E}^{A}\right)$ such that $u_{n}>0$ for $n \geq N$. Let $L: S \rightarrow \mathbb{R}$ be the functional on $S$ defined by $L(x)=\mathscr{L}\left(x_{n}, u_{n}\right)$. The set

$$
K=\{x \in S: L(x)=0\}
$$


is the kernel of linear functional $L$ defined on $S$. Then $x \in K$ satisfies

$$
u_{n+1} x_{n+1}^{[2]}-u_{n}^{[1]} x_{n+1}^{[1]}+u_{n}^{[2]} x_{n+1}=0 .
$$

Multiplying the last equation by $\left(u_{n+1} u_{n+2}\right)^{-1}$, we get

$$
\frac{u_{n+1} x_{n+1}^{[2]}-u_{n+1}^{[1]} x_{n+1}^{[1]}}{u_{n+1} u_{n+2}}+\frac{u_{n+1}^{[1]} x_{n+1}^{[1]}-u_{n}^{[1]} x_{n+1}^{[1]}}{u_{n+1} u_{n+2}}+\frac{u_{n}^{[2]} x_{n+1}}{u_{n+1} u_{n+2}}=0 .
$$

From here and using

$$
\begin{aligned}
p_{n+1} \Delta\left(\frac{x_{n+1}^{[1]}}{u_{n+1}}\right) & =\frac{u_{n+1} p_{n+1} \Delta x_{n+1}^{[1]}-x_{n+1}^{[1]} p_{n+1} \Delta u_{n+1}}{u_{n+1} u_{n+2}} \\
& =\frac{u_{n+1} x_{n+1}^{[2]}-u_{n+1}^{[1]} x_{n+1}^{[1]}}{u_{n+1} u_{n+2}}
\end{aligned}
$$

we obtain

$$
p_{n+1} \Delta\left(\frac{x_{n+1}^{[1]}}{u_{n+1}}\right)+\frac{x_{n+1}^{[1]} \Delta u_{n}^{[1]}}{u_{n+1} u_{n+2}}+\frac{u_{n}^{[2]} x_{n+1}}{u_{n+1} u_{n+2}}=0 .
$$

In view of the identity

$$
x_{n+1}^{[1]} \Delta u_{n}^{[1]}+u_{n}^{[2]} x_{n+1}=u_{n}^{[2]} \Delta x_{n+1}+u_{n}^{[2]} x_{n+1}=u_{n}^{[2]} x_{n+2},
$$

(4.13) can be rewritten in the form (4.7). Since $\operatorname{dim} K=\operatorname{dim} S-1=2$, we get the conclusion.

Claim (b). Let $x$ be a fixed nonoscillatory solution of (E) such that $x_{n}>0$ for $n \geq N$. Let $L^{\star}: S^{\star} \rightarrow \mathbb{R}$ be the functional on $S$ defined by $L^{\star}(u)=\mathscr{L}\left(x_{n}, u_{n}\right)$. The set

$$
K^{\star}=\left\{u \in S^{\star}: L^{\star}(u)=0\right\}
$$

is the kernel of linear functional defined on $S^{\star}$. Then $u \in K^{\star}$ satisfies

$$
x_{n+1} u_{n}^{[2]}-x_{n+1}^{[1]} u_{n}^{[1]}+x_{n+1}^{[2]} u_{n+1}=0 .
$$

Multiplying the last equation by $\left(x_{n+1} x_{n+2}\right)^{-1}$, we get

$$
\frac{x_{n+1} u_{n}^{[2]}-x_{n+1}^{[1]} u_{n}^{[1]}}{x_{n+1} x_{n+2}}+\frac{x_{n+1}^{[2]} u_{n+1}}{x_{n+1} x_{n+2}}=0 .
$$

From here using

$$
\begin{aligned}
r_{n+1} \Delta\left(\frac{u_{n}^{[1]}}{x_{n+1}}\right) & =\frac{x_{n+1} r_{n+1} \Delta u_{n}^{[1]}-u_{n}^{[1]} r_{n+1} \Delta x_{n+1}}{x_{n+1} x_{n+2}} \\
& =\frac{x_{n+1} u_{n}^{[2]}-x_{n+1}^{[1]} u_{n}^{[1]}}{x_{n+1} x_{n+2}}
\end{aligned}
$$

we get (4.8). Since $\operatorname{dim} K^{\star}=\operatorname{dim} S^{\star}-1=2$, we get the conclusion. 
Corollary 4.5. (a) If (E) is oscillatory, then there exists a basis for $S$ consisting of one nonoscillatory solution and two oscillatory solutions.

(b) If $\left(E^{A}\right)$ is oscillatory, then there exists a basis for $S^{\star}$ consisting of one nonoscillatory solution and two oscillatory solutions.

Proof. By Theorem 3.3 and Lemma 3.1, there exist Kneser solution of (E) and strongly monotone solution of $\left(\mathrm{E}^{A}\right)$. Assume that $(\mathrm{E})$ and $\left(\mathrm{E}^{A}\right)$ are oscillatory. In view of Theorem 4.4 and its proof, (E) has two independent solutions which must be oscillatory. Similarly, there exist two independent oscillatory solutions of $\left(\mathrm{E}^{A}\right)$. Because $\operatorname{dim} S=\operatorname{dim} S^{\star}=3$, the proof is complete.

THEOREM 4.6. (a) If (4.7), where $u$ is a nonoscillatory solution of $\left(E^{A}\right)$, is oscillatory, then (E) and $\left(E^{A}\right)$ are oscillatory.

(b) If (4.8), where $x$ is a nonoscillatory solution of $(E)$, is oscillatory, then (E) and $\left(E^{A}\right)$ are oscillatory.

Proof. Assume that (4.7) is oscillatory, that is, there exists an oscillatory solution $x$ of (4.7). Using the same argument as in the proof of Theorem 4.4, we obtain that $x \in K$, where $K$ is defined by (4.9). Hence $x$ is an oscillatory solution of (E). By Theorem 4.3, $\left(\mathrm{E}^{A}\right)$ is oscillatory, too.

Similarly, if $u$ is an oscillatory solution of (4.8), then $u \in K^{\star}$. Hence $u$ is oscillatory solution of $\left(\mathrm{E}^{A}\right)$ and this implies that $(\mathrm{E})$ is oscillatory too.

Theorem 4.7. Equation (E) is oscillatory if and only if (4.8) is oscillatory.

Proof. Assume that (E) is oscillatory, that is, there exists an oscillatory solution $y$ of $(\mathrm{E})$. By Theorem 3.3, there exists a Kneser solution $x$ of (E). According to (2.27),

$$
0=\left|\begin{array}{ccc}
x_{n} & y_{n} & x_{n} \\
x_{n}^{[1]} & y_{n}^{[1]} & x_{n}^{[1]} \\
x_{n}^{[2]} & y_{n}^{[2]} & x_{n}^{[2]}
\end{array}\right|=\mathscr{L}\left(x_{n-1}, C_{n-1}\right),
$$

where $C$ is defined by (2.1). By Theorem 2.1, $C$ is solution of $\left(\mathrm{E}^{A}\right)$. From Lemma 4.2 follows that $C$ is an oscillatory solution of $\left(\mathrm{E}^{A}\right)$. In view of Lemma 2.4,

$$
L^{\star}(C)=\mathscr{L}\left(x_{n}, C_{n}\right)=0,
$$

hence $C \in K^{\star}$, where $K^{\star}$ is defined by (4.15). From here and the proof of Theorem 4.4, we get the fact that $C$ is an oscillatory solution of (4.8). The opposite statement follows from Theorem 4.6.

Open problems.

(1) It is an open problem whether the existence of an oscillatory solution of $\left(\mathrm{E}^{A}\right) \mathrm{im}$ plies the oscillation of (4.7). To solve this problem, it would be useful to find the functional $\mathscr{L}^{\star}$ defined on $S^{\star} \times S$ with similar properties to those of $\mathscr{L}$ described in Lemmas 2.4 and 2.5 .

(2) To generalize results of this paper to the linear $n$ th-order difference equations involving quasi-differences. 


\section{Acknowledgment}

This work was supported by the Czech Grant Agency, Grant 201/04/0586.

\section{References}

[1] R. P. Agarwal, Difference Equations and Inequalities, 2nd ed., Monographs and Textbooks in Pure and Applied Mathematics, vol. 228, Marcel Dekker, New York, 2000.

[2] M. Cecchi, Z. Došlá, and M. Marini, An equivalence theorem on properties A, B for third order differential equations, Annali di Matematica Pura ed Applicata. Series IV 173 (1997), 373-389.

[3] Z. Došlá and A. Kobza, Global asymptotic properties of third-order difference equations, Computers \& Mathematics with Applications. An International Journal 48 (2004), no. 1-2, 191-200.

[4] _ On nonoscillatory solutions of third order difference equations, Proceedings of the Eighth International Conference of Difference Equations and Applications (S. Elaydi, G. Ladas, B. Aulbach, and O. Dosly, eds.), Taylor \& Francis, Chapman \& Hall/CRC, Boca Raton, Fla, 2005, pp. 105-112.

[5] U. Elias, Oscillation Theory of Two-Term Differential Equations, Mathematics and Its Applications, vol. 396, Kluwer Academic, Dordrecht, 1997.

[6] M. Migda, Nonoscillatory solutions of some higher order difference equations, Colloquium on Differential and Difference Equations, CDDE 2002 (Brno), Folia Fac. Sci. Natur. Univ. Masaryk. Brun. Math., vol. 13, Masaryk University, Brno, 2003, pp. 177-184.

[7] J. Popenda and E. Schmeidel, Nonoscillatory solutions of third order difference equations, Portugaliae Mathematica 49 (1992), no. 2, 233-239.

[8] B. Smith, Oscillatory and asymptotic behavior in certain third order difference equations, The Rocky Mountain Journal of Mathematics 17 (1987), no. 3, 597-606.

[9] __ Oscillation and nonoscillation theorems for third order quasi-adjoint difference equations, Portugaliae Mathematica 45 (1988), no. 3, 229-243.

[10] Linear third-order difference equations: oscillatory and asymptotic behavior, The Rocky Mountain Journal of Mathematics 22 (1992), no. 4, 1559-1564.

[11] P. J. Y. Wong and R. P. Agarwal, Nonoscillatory solutions of functional difference equations involving quasi-differences, Fako de l'Funkcialaj Ekvacioj Japana Matematika Societo. Funkcialaj Ekvacioj. Serio Internacia 42 (1999), no. 3, 389-412.

Zuzana Došlá: Department of Mathematics, Masaryk University, Janáčkovo nám. 2a, 60200 Brno, Czech Republic

E-mail address:dosla@math.muni.cz

Aleš Kobza: Department of Mathematics, Masaryk University, Janáčkovo nám. 2a, 60200 Brno, Czech Republic

E-mail address: akob@math.muni.cz 